\title{
La Influencia del Mariscal Santa Cruz en el Proceso Educacional del Perú.
}

Una década, intensamente vivida, dura en nuestro país la acción y la influencia, positiva unas veces, negativa otras, pero existente siempre, del Mariscal Andrés Santa Cruz quien ocupa también un lugar, no subalterno, en la historia de la pedagogía peruana.

Todavía durante la permanencia de Bolívar en el Perú, y por renuncia del General La Mar, asumió Santa Cruz la presidencia del Conseio de Estado y afrontó los embates de la reacción antibolivariana que produjo hondas agitaciones públicas, el déstierro de Euna Pizarro," del general $\mathrm{Ne}$ cochea, del Vice-Almirante Guisse y de otros hombres eminentes, varios fusilamientos entre ellos el del teniente Aristizábal que fué el que provocó más protestas, y algunas sublevaciones en Tacna, Ica y Camaná. Bajo la fuerte presión de las autoridades políticas, cincuentinueve colegios electorales, con excepción del de Tarapacá, aprobaron la constitución vitalicia y el propio Santa Cruz, quebrantando la ley que ordenaba la revisión de esas actas electorales por la Corte Suprema, en acto dictatorial, declaró en vigencia 1a Constitución bolivariana y la hizo jurar solemnemente el 9 de diciembre de 1826 , segundo aniversario de la batalla 
de Ayacucho, nombró a Bolivar Presidente del Perú y suprimió los municipios. El 26 de enero siguiente estalló un motín militar, patrocinado por Don Manuel Lorenzo Vidaurre, Presidente de la Corte Suprema, desconociendo la Carta Vitalicia y apresando a los principales jefes de las tropas colombianas que sostenian a la Junta de Gobierno. El Cabildo de Lima, reunido al siguiente día, protestó también contra la Constitución vitalicia y envió una comisión a Santa Cruz, que entonces se encontraba en Chorrillos, para que asumiera interinamente la Presidencia de la República y pusiera en vigencia la Carta Politica de I823. Simultáneamente llegaba una carta de Bolivar renunciando a toda participación en la política peruana. Santa Cruz, en vista de ello, aceptó la présidencia el 28 de enero de ¥827, lanzó un Manifiesto a la Nación derogando la Carta Vitalicia y licenció a las tropas colombianas que regresaron a su país.

Las agitaciones públicas que sobresaltaban por igual a los gobernantés yia los gobernados, no permitieron a los primeros dedicarse íntegramente ah progreso de la instrucción pública. Díctanse, sin embargo, en este periodo tur-bulento, algunas importantes medidas dignas de mención. El Consejo de Estado, por decreto del 23 de abril de I825, refrendado por Hipólito Unánue, J. M. Pando y T. Heres, reglamentó la Dirección General de Estudios que había sido creada por la Constitución de 1823 en su art. I85. Dispuso el citado decreto que la Dirección General de Estucios, que debía funcionar en la capital de la República, estaría integrada por el Rector de la Universidad Mayor de San Marcos, los rectores de los demás colegios de la ciudad-San Carlos, Santo Toribio e Independencia (San Fernando) y el Decano del Colegio de Abogados (art. 
$\left.2 .^{9}\right)$. Dependian de este organismo las Direcciones que debian crearse en las distintas capitales de los departamentos para supervigilar la marcha de la enseñanza, inclusive la economía de los planteles y proponer las reformas necesarias para su mejor desenvolvimiento; y los inspectores encargados de la vigilancia de las escuelas de primeras letras, aulas de latinidad y enseñanza de ciencias. La labor de la Dirección General consistía en reunir y aportar los materiales para los planes y reglamentos de estudios cuya formación correspondía al Congreso de conformidad con el art. 183 de la Constitución de 1823 (I).

Todavía bajo la presidencia de La Mar, el Consejo de Gobierno expidió algunos decretos importantes, relacionados con la educación pública, entre otros el otorgamiento de una beca en el Colegio de la Independencia, la fundación de escuelas primarias para niñas y la invitación a quienes se creyeran con aptitudes para desempeñar el cargo de directores o maestros de las mismas (2), primer anticipo en la República, de los concursos magisteriales; reorganizando y restaurando el "Colegio de Indigenas "La Libertad", denominado anteriormente del Príncipe, por los servicios que estos prestaron a la causa de la independencia (3); creando una academia de taquigrafía en la Universidad de San Marcos y enviando a cuatro jóvenes becarios a estudiar a Inglaterra por cuenta del Gobierno (4); prohibiendo el ingreso al país de libros que atentasen contra la moral y la educación de la juventud, fijando las penas y

(1) "Gaceta del Gobierno", T. VII, N.9 39, del 1.0 de mayo de 1825 Oviedo T. IX. L. III.

(2) "Gaceta del Gobierno", T. VII, N.o 40, del jueves 5 de mayo de 1825.

(3) Ibid. Tomo VII, N.o 46, del jueves 19 de mayo de 1825.

(4) Ib. T. VIII, N.e 5, del 17 julio de 1825. 
multas a los infractores (5); reorganizando las escuelas de primeras letras, las que estarían a cargo de religiosos "de probidad y amor al bien público" y a quienes se le asignaron las pensiones correspondientes (6); y estableciendo en el Colegio de Santo Tomás una escuela central normal para jóvenes, conforme al método lancasteriano, y ordenando que en el citado colegio se dictaran gratuitamente clases de Filosofía y Teología ( 7 ).

Por decreto del 20 de setiembre de 1825 , refrendado por Hipólito Unánue, se creó el Convictorio de Bolivar, refundiendo en el nuevo plantel los Colegios de San Carlos y del Príncipe. Su reglamentación la expidió Santa Cruz, Presidente del Consejo de Gobierno, el 26 de octubre de I826. Comprendía la enseñanza los estudios de Lengua y Literatura Clásicas (Latina y Griega), Filosofía, Metafísica y Lógica, Derecho Natural y de Gentes, Derecho Público y Constitucional, Derecho Canónico, Derecho Romano, Derecho Patrio Civil y Criminal, Matemáticas, Económía Política, Cronologia e Historia. Predominaban los estudios jurídičs. Seucrearon (doce becas para indígenas y se le señaló al plantel la renta respectiva para su sosteni-
miento (8).

Fué también importante el decreto del Consejo de Gobierno expedido por esta misma época, estableciendo tres aulas de humanidades: la primera de Lengua Castellana, la segunda de Gramática Latina hasta la sintaxis y la tercera de Poesía y Retórica. Junto con la designación del lo-

(5) Tb. T. VIII, N.o 10, del 4 de agosto de 1825.

(6) Ib. T. VIII, N.o 14 del jueves 18 de agosto de 1825

(7) Ib. T. VIII, N.9 16, del jueves 25 de agosto de 1825.

(8) Estuvo en vigencia este decreto hasta el 13 de noviembre de 1829 en que fué derogado por ley que estableció el régimen anterior. Registro
Oficial de la República Peruana, 1829. 
cal y de la renta respectiva, dispúsose, además, para el mejor desempeño de la docencia, que a los maestros se les proporcionara vivienda en el mismo local (9). No se ha reparado hasta ahora en la importancia de esta disposición gubernativa que constituye uno de los antecedentes de la política educacional perfeccionada más tarde en nuestro país tendiente a favorecer la construcción de casas para los maestros. Ella aspiró a realizar, además, uno de los consejos pedagógicos de Locke, el reformador de las escuelas inglesas del siglo XVII, en el sentido de que los maestros deberían hacer, hasta donde le fuera posible, la misma vida de sus alumnos.

La enseñanza del latín, la lengua madre aunque muerta, se complementó entonces con el aprendizaje de las lenguas vivas, al que desde los albores de la República se le concedió, por lo menos en teoría, un interés particular. En la Bibliotcca Nacional se abrió, con-ese objeto, en setiembre de I825, un aula para la enseñanza del inglés y del francés (IO).

El Colegio de Santo Tomás amplió su enseñanza con la apertura de tres nuevas aulas, la de Filosofía, la de Teología Dogmática y la de Derecho Canónico, disponiéndose además la gratuidad de la enseñanza de estos cursos y otorgándoseles especiales facilidades a los alumnos, entre otras la de concederles habitación en el colegio a quienes viviesen lejos de la Capital (II). Se creó, por decreto del Consejo, refrendado por Unánue, Salazar, La-

\footnotetext{
bre, 1829 .

(9) "Gaceta del Gobierno", T. VIII, N.8 24, del jueves 22 de setiem-

(10) Ibidem. T. VIII. N.o 26, del jueves 29 de setiembre de 1825.

(11).- Nota del Rector del Colegio de Santo Tomás al Ministro de Estado del Departamento de Gobierno.- "Gaceta del Gobierno", tomo VIII, N.P 28, del juevos 6 de octubre de 1825 .
} 
rrea y Loredo, expedido el 6 de octubre de I825, un Gineceo o escuela educacional para mujeres, que constaba de dos aulas, enseñándose en la primera, religión cristiana, escritura y principios de aritmética; y en la segunda "labores propias de una madre de familia" y además música, geografía e historia (I2). Se ordenó la reapertura del Seminario Conciliar de Santo Toribio, cuyo plan de enseñanza, "de acuerdo con los adelantos del siglo" debía ser elaborado por la Dirección General de Estudios, el cual sería presentado al Consejo de Gobierno "para su aprobación o rechazo" (I3). El Consejo envió, por cuenta del Estado, a estudiar a Inglaterra a seis jóvenes: uno del departamento de Lima, otro del de La Libertad, dos del Cuzco y dos de Arequipa (I/4). Se fusionaron en Ayacucho la Universidad de San Cristóbal de Huamanga con el Seminario del mismo nombre denominándose en lo sucesivo "Colegio, Seminario y Universidad de San Cristóball", habiéndose encargado a la Dirección de Estudios Departamental la formulación de los planos respectivos, la asignación de süsrentas, el nulmero de becas, etc. ( I 5). Se anotan algunos progresos en el Colegio de la Independencia al que concurren numerosos alumnos y en el que se enseña Humanidades, Matemáticas, Geografía, Química, Anatomía, Medicina y Dibujo, anunciándose la próxima aper-
tura de nuevas facultades ( 6 ).

(12) "Gaceta del Gobierno", tomo VIII, N.9 29, del domingo 9 de octubre

(13) Ibidem. T. VIII. N.o 33, del domingo 23 de octubre de 1.825.

(14) Tbidem. T. VIII. N..$^{\circ} 38$, del jueves 10 de noviembre de 1825 . frendado por Unanue, Salazar, Larrea y Loredo.- "Gaceta de 1825 y reTomo VIII. - N.o 40. (16) Nota del Rector del Colegio de la Independencia al Sr. Ministro de
Gobierno.- "Gaceta del Gobierno". T. VIII.-N.9 42, 
Santa Cruz procedió en I\$26 a reglamentar las escuelas lancasterianas que funcionaban muy deficientemente. Dos nuevas fueron creadas en Lima: la de Santo Tomás para varones y la de la Concepción para niñas.

En su deseo de impulsar la difusión de la cultura, mediante el conocimiento de las obras útiles, tendientes a ese fin, se publicó en el mes de abril de I\$26 el libro titulado "De la influencia de los diferentes climas del Universo sobre el hombre y en particular de la influencia de los climas de América Meridional" cuyo autor fué el doctor don Abel Victorino Braudin, doctor en Medicina, Caballero, de la Orden Real de la Legión de Honor de Francia (I7). Esta obra constituye uno de los antecedentes en los estudios de la Sociología Peruana y le fué de notoria utilidad al sabio ariqueño don Hipólito Unánue en sus investigaciones científicas sobre el clima de Lima.

No descuidó tampoco el Consejo de Gobierno recomendar a los prefectos que establecieran, en sus respectivas jurisdicciones, dos escuelas lancasterianas de instrucción primaria, una paraniños y otra para niñas (I8).

.El Mariscal Santa Cruz, por decreto expedido el 4 de febrero de $\mathrm{I} 827$, ordenó la creación del "Colegio General de Ciencias y Artes de la Independencia Americana" que empezó a funcî́nar el I5 de junio de ese mismo año. Tal medida constituyó el tercer y último paso para la funda1826.

(17) "Gaceta del Gobierno", T. IX, N.P 31 del sábado 15 de abril de 1826 .

(18) "El Peruano", Semestre I.-N.o 48, miérecoles 15 de noviembre de 
ción de la Universidad de Arequipa. El primer paso había sido la creación del Seminario de San Jerónimo y el segundo el establecimiento de la Academia Lauretana.

Un año atrás, en I826, el Prefecto de Arequipa General Antonio Gutiérrez de La Fuente, vivamente interesado en el progreso intelectual de su ciudad natal, inició sus gestiones para fundar un colegio de ciencias y artes, señalando desde entonces como local el convento de los agustinos quienes movieron toda clase de influencia para no cederlo, encontrando al principio el apoyo del Ministro Pando. Secundaron al Prefecto los miembros de la Dirección Departamental de Estudios, integrada en Arequipa por el Deán Manuel Fernández de Córdova, Fr. Gualberto Valdivia "máximo exponente del carácter combativo y del fervor idealista de Arequipa de aquella época" (I9), el Dr. Mariano Ugarte, Vocal de la Corte Superior y el Dr. Manuel Cayetano Loyo, Secretario de la Prefectura. Estos, en unión de don Mariano Rivero, miembro de la Dirección General de Estudios, quien, se encontraba por aquel entonces en Arequipa, redactaron el plan orgánico del nuevo colegio, dándole como base la Academia Lauretana con sus rentas, alumnos y catedráticos e indicando las asignaturas que se enseñarian: lenguas latina, castellana, francesa e inglesa; derechos natural, de gentes, público, patrio, canónico, matemáticas elementales y aplicadas, astronomía, química, física, mineralogía, geología, botánica, teología, anatomía, cirugía, medicina, economía política, agricultura

(19) Edilberto Zegarra Ballon Valdez, "La Universidad de Arequipa a través de cien años".-Discurso de Orden leído en la sesión solemne para con. San Agustín. 
y dibujo. Los primeros años de su existencia fueron accidentados y sus reveses continuos (20).

Al inaugurar el plantel el Prefecto Gutiérrez de la Fuente dirigió una vibrante alocución a los profesores. "A vosotros-les dijo-les entrega el Gobierno la juventud de este departamento. Sembrad en ella la pura moral, la ciencia de amar su raza y sus semejantes, la ciencia de vivir en sociedad, la ciencia de engrandecerse y que las $\mathrm{fu}^{-}$ turas generaciones en medio de su opulencia os bendigan con gratitud y admiración. No cuicéis cardar en molleras añejas habitudes que presto terminarán en el polvo. La juventud que os presento es vuestro campo: cultivad en ella. y responded a la Patria de la obligación que os impongo este memorable día".

Un mes antes, el 2 de junio de 1827 , el Prefecto, acogiendo y haciendo suya una iniciativa de la Dirección Departamental de Estudios, en el mismo decreto de nombramiento de profesores, declaró "establecida en dicho colegio la Universidad bajo el nombre titular del Gran Padre de la Iglesia San Agustín'" Debían componer la Universidad los doctores existentes en Arequipa por orden de antigüedad, los catedráticos del Colegio de la Independencia, los socios de número de la Academia Lauretana, quienes tendrían el derecho de graduarse sin pensión alguna, previas las solemnidades que se establezcan; y los doctores de las otras

(20) Il primer euerpo de profesores lo integraron Santiago Ophelau, Rafael Evaristo Barriga en Lengua Castellana, el presbitero Juan Gualberto Valdivia en Filosofía y Matemática, el Licenciado Andrés Martínez en Dorecho Civil, Juan María Corbacho en Bellas Artes, Manuel Amat y León, en Economía Política, Dr. Mariano Odriozola en Anatomía y Cirugía, Dr. Leonardo Navas en Medicina, Tadeo Chávez en Derecho Natural, de Gentes e Internacional, Pedro Jiménez Abril en música y Juan Manuel de Recavarren 
Universidades de la República o de América que estuviesen o pudiesen estar en Arequipa quienes "tendrán asiento en el claustro y gozarán de todas las preeminencias de honor que disfrutan los de aquí". El uniforme para los alumnos era el vestido negro y sombrero redondo y el distintivo de una medalla de plata al costado izquierdo. Los profesores $y$ catedráticos, igual vestido con banda celeste, escudo y sombrero armado (2I).

La Universidad se inauguró solemnemente el II de noviembre de 1828 . "Tengo-dijo en la ceremonia inaugural el Prefecto General Gutiérrez de la Fuente-el inefable placer de cerrar la administración de mis negocios públicos, abriendo las puertas de este establecimiento interesante". Gobernaba a la sazón el pais el Gran Mariscal Don Agustín Gamarra. La Universidad entró en funciones inmediatamente, limitando sus actividades a la colación de grados académicos de bachiller, licenciado y doctor. Fué reconocida oficialmente solo el 6 de mayo de 1835 , por decreto supremo quécexpidió ceb Presidente provisorio de la República, general Luis José de Orbegoso, quien se encontraba entonces en Arequipa.

La apertura de algunos Colegios Nacionales demostró entonces el interés de los poderes públicos por la instrucción. Aparte del Colegio Nacional de la Independencia Americana de Arequipa, cuya importancia ya hemos puntualizado, se abrieron otros planteles de la misma índole. El I. ${ }^{\circ}$ de junio de 1827 abrió sus puertas, con quince alum(21) Plan de la Universidad, aprobado por el Prefecto Gutiérrez de la
Fuente el 2 de junio de 1827. 
nos, el Colegio Nacional de San Luis Gonzaga en Ica, precisamente al año justo de su creación por decreto supremo, destinándosele al principio a la enseñanza de jurisprudencia, filosofía y matemáticas. En I827 el Colegio Nacional de Ciencias del Cuzco, que se había trasladado al local de la Compañía de Jesús, amplió su docencia con las nuevas cátedras de Medicina, Derecho Civil y Derecho Canónico. En I833 tenía quince cátedras bien dotadas, con sus talleres de carpintería y herrería, con más de cien alumnos internos "que solo usaban servicio de plata". Por ley del 4 de marzo de 1828 se creó el Colegio de la Virtud Peruana en la ciudad de Huánuco, capital entonces del departamento de Junín, plantel que más tarde fué transformado en Escuela Central de Minería por decreto del 8 de julio de 1846 y reorganizado nuevamente en I 848 como Colegio de Minería đictándose los cursos de Lógica, Metafísica, Latín, Geometría y Cálculo. El Colegio de La Libertad en Huaraz fué creado por ley del zo de cenerode 1828 y funcionó en el Convento supresaj de San Francisconnespués de no pocas y esforzadas gestiones de los hombres notables del lugar, se creó, por decreto del II de noviembre de I829, el Colegio Nacional de San Ramón, instalado solamente el 8 de setiembre de I83I. Ocurrió lo propio con el Colegio Nacional de San Carlos de Puno, creado por Resolución Legislativa del I7 de diciembre de I829 y que comenzó a funcionar el 16 de abril del año siguiente con el nombre de Colegio de Ciencias Naturales. Por ley del I4 de junio de I83i se fundó en la villa de Huancavelica el Colegio Nacional de Ciencias y Artes con el nombre de "Victoria de Ayacucho", que funcionó a partir del I7 de marzo de 1833 , en el convento supreso de Santo Domingo, 
con sólo tres cátedras, Gramática Castellana y Latina, Filosofía, Matemáticas y Mineralogía, las que fueron ampliadas posteriormente, en 1833 , con las cátedras de Derecho Natural, Internacional, Romano, Civil y Canónico. El Colegio Nacional de San Ramón de Ayacucho fué creado por ley del I 4 de julio de 183 I siendo los primeros alumnos becarios de todas las provincias. La resolución suprema del 22 de diciembre de 1832 creó el Colegio Nacional de San José de Chiclayo que sólo purlo funcionar 26 años más tarde con tres cátedras: una de Matemáticas Puras y Geografía, otra de Filosofía e Historia y la tercera de Latín, Gramática Castellana y Religión. En $18_{33}$ empezó a funcionar el Colegio de San Miguel de Piura, creado por decreto supremo de mayo de I827 con el nombre de Colegio del Carmen.

La acción legislativa abarcó también la educación femenina, creando para tal efecto un Colegio de Educandas en Ica, por ley del 24 de mayo de I828-que fué derogada el Io de octubre de I869-; el Colegio del Espíritu Santo, para niñas, en Lima, por ley del i9 de julio de 1830 , reglamentada el 24 de marzo de 1849 . Constaba este último plantel de dos departamentos, uno gratuito y otro para pensionistas, enseñándose en él las labores y ciencias do-
mésticas.

La Constitución de I828 mantiene entre las atribuciones del Congreso la de "formar planes generales de edu. cación e instrucción pública y promover el adelantamiento 
de las artes y las ciencias" (art. 48). Otorga a las Juntas Departamentales la facultad de "promover la educación e instrucción pública, conforme a los planes aprobados por el Congreso" (art. 75, inc. $2 .^{\circ}$ ). Y, ampliando los dispositivos, "garantiza también la instrucción primaria gratuita a todos los ciudadanos; la de los establecimientos en que se enseñen las ciencias, literatura y artes; la inviolabilidad de las propiedades intelectuales y los establecimientos de piedad y beneficencia" (art. I7I). Bello enunciado doctrinario, que revela los propósitos del legislador, esta garantía constitucional queda sin cumplirse a causa de las agitaciones políticas que perturbaron por esta época el ritmo normal en la marcha del país.

Derrocado La Mar, el Congreso reunido el $3^{\mathrm{I}}$ de agosto cle I829, nombró como Presidente Provisorio al Mariscal Gamarra, ratificado en el cargo supremo, con el carácter de Presidente Constitucional, por las elecciones populares realizadas en diciembre de ese mismo año. Su gobierno afrontó graves complicaciones internacionales e internas, provenientes las primeras de las pretensiones de Santa Cruz, a la sazón "gobernante de Bolivia, de anexarse a ese país el territorio peruano de Arica; y las segundas, de catorce movimientos revolucionarios que estallaron sucesivamente contra Gamarra, quien para atender personalmente a la conservación del orden público y a la estabilidad de su régimen político, marchó al sur, al frente de sus tropas, dejando el mando supremo al Vice-Presidente de la República, General Antonio Gutiérrez de la Fuente.

En materia educacional el Vice-Presidente de la República expidió el I9 de noviembre de I830, una resolución refrendada por el Ministro Pedemonte, declarando en vigencia el decreto protectoral de San Martín, firmado el 24 de noviembre de $\mathrm{I} 82 \mathrm{I}$ relativo a la educación de los hijos 
de madres esclavas, nacidos después de la independencia, considerando que él no estaba en oposición con el espíritu de la Carta Política en vigor (22).

La situación política se enturbió más y más por las rivalidades entre el Jefe del 'Estado y su Vice-Presidente, a quien se enfrentó, con varonil energía, la esposa del Presidente, “ la Mariscala”. El 9 de enero de I83I Gutiérrez de la Fuente resolvió trasladar la sede del gobierno al vecino puerto del Callao, so pretexto de "aprovechar la estación veraniega". Para impedirlo, la Mariscala lanzó a las turbas asalariadas que asaltaron la casa del Vice-Presiclente en Lima, obligándolo a fugar al Callao y embarcarse en la fragata inglesa "Saint Lewis". Asumió, mientras tanto, el mando supremo el ciudadano Don Andrés Reyes, Presidente del Senado.

El encargado del mando supremo, mientras clurase la ausencia de Gamarra, expidió un decreto dotando al Colegio de Ciencias de Ica de dos cátedras y asignándole una renta de ochocientos pesos añuales.2Dispuso, además, que igual cantidad se diese a los colegios de las demás provincias $(23)$.

La carencia de una auténtica orientación pedagógica, la muy deficiente administración de las rentas escolares y las frecuentes convulsiones políticas explican el lamentable atraso de la instrucción pública en esta época. Se dejaba sentir la urgente necesidad de una reglamentación orgánica y ella se intentó por el decreto del is de noviembre ce I833 que, por los puntos que contiene, bien puede considerarse como un esbozo del Reglamento de Instrucción Pú1830.

(22) "Peruano del Sud", Tomo II, N. 5, del sábado 4 de diciembre,

(23) Tbidem. Tomo II, N.o 33, del 30 de julio de 1831. 
blica del Perú. Tendiendo a la uniformidad de la enseñanza, que por entonces era de lo más inconexa y heterogénea, ei decreto de 1833 determina las materias de aprendizaje obligatorio, a saber: lectura, escritura. matemáticas elementales, gramática castellana, religión y costura para las niñas; crea el Departamento de Instrucción Primaria encargándolo de la administración económica y de la dirección pedagógica de las escuelas primarias de esta capital, actividades que hasta entonces eran incumbencia del $\mathrm{Mu}-$ nicipio la primera y del Poder Ejecutivo la segunda (24); mantiene el método lancasteriano; prohibe a los maestros recibir ningún pago por parte de los alumnos; implanta el régimen de los exámenes semestrales, uno en privado $\mathrm{y}$ otro en público para acreditar el aprovechamiento de los estudiantes; bonifica a los preceptores que sobresalgan en el desempeño de sus funciones para estimularlos en las mismas; prohibe la coeducación, a fin de que en un mismo plantel y en las mismas aulas no se enseñe simultáneamente a hombres y mujeres éstablece el horariosde las labores dlarias de 9 a $\mathbf{I} 2$ m. y de 3 cais pling ary premia a los alumnos más aprovechados con medallas de oro y plata.

La Convención Nacional, retunida en 1834 , expidió ese mismo año la Constitución Política que mantiene, en lo referente a la educación pública, los mismos principios consagrados en las Constituciones anteriores. Correspondía siempre al Congreso "formar planes generales de edu-

(24) El nuevo eargo de Director de. Instrucción Primaria se confí al presbitero Navarrete. 
cación e instrucción pública para los establecimientos dotados de fondos nacionales" (art. $5 \mathrm{I}$, inc. I6). Declárase igualmente que "la instrucción primaria es gratuita para todos los ciudadanos; y también la científica en las capitales o en el lugar más a propósito de cada departamento". (art. I I I).

La gratuidad de la enseñanza primaria para todos los ciudadanos no pasaba de ser un laudable aunque incumplido propósito de los legisladores. Lo propio decimos de la enseñanza científica en cada departamento, pese a este dispositivo constitucional y a una anterior circular, suscrita el I9 de octubre de 183 I por el Ministro doctor Matías León, declarando la gratuidad de la segunda enseñanza.

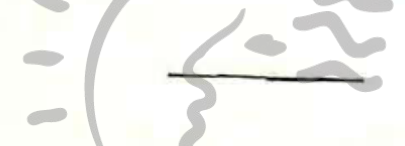

La Confederación Perí Boliviana, implantada a sangre y fuego por Santa Cruz en 1836 , combatida a sangre y fuego por las expediciones peruano-chilenas y derrocada a sangre y fuego por las mismas en I 839 , abarca uno de los trienios más turbulentos de nuestra historia republicana. Es de admirar, por lo mismo, como en medio de tan graves preocupaciones de orden interno e internacional, puco el Protector Santa Cruz emprender un laudable plan de reformas educacionales, época de transición entre este primer período de la desorganización inicial de la pedagogía peruana que comprende el ciclo I82I-I850 y la era en que principian, con el Mariscal Castilla, los intentos de sistematización educativa.

Las reformas pedagógicas de Santa Cruz abordaron dos cuestiones importantes: la transformación de los estu- 
dios en el Colegio de San Carlos y la creación de las administraciones departamentales para las rentas de enseñanza y beneficencia.

El Convictorio Carolino, que tan brillante actuación había tenido en las postrimerias del Virreynato, ampliando las perspectivas de la cultura peruana, se había dedicado preferentemente en los albores de la República a la enseñanza del derecho. Santa Cruz comprendió la necesidad de reorganizarlo, adaptáncolo a las condiciones sociales en las que vivía el pais y a las exigencias espirituales que demandaban un mayor incremento en la instrucción pública. El Protector amplió, por eso, la órbita educacional del Colegio de San Carlos con la enseñanza de las ciencias físicas y naturales, desarrollándose el plan de estudios en ocho años: los cuatro primeros dedicados a los conocimientos generales tanto científicos como literarios y los cuatro últimos dedicados al estudio del derecho. Es importante esta reorganización de Santa Cruz porque, si bien es cierto que a pesar de ella permanece el Convictorio con su estructura híbrida, conlloslcaracterese propios de las Facultades Universitarias ye de los colegios de segunda enseñanza, defecto generalizado en esta época, en cambio ya se inicia la diferenciación en los dos ciclos cuatrienales consecutivos: el primero de ellos corresponde a la enseñanza secundaria y el segundo a los estudios académicos. Santa Cruz dá así el primer paso de una reforma pedagógica que va a complementarse y perfeccionarse posteriormente.

Abordó también el Mariscal el problema económico de la enseñanza, creando las Administraciones Departamentales para el incremento de sus rentas y el control de las mismas, debiendo además supervigilar que los municipios y las beneficencias contribuyeran al sostenimiento de 
las escuelas en la proporción a que estaban obligados. E1 funcionamiento de estos organismos fué de corta duración. Desaparecieron las Administraciones Departamentales a la caída de la Confederación Peruano-Boliviana, después de la derrota de Yungay, a raíz de la cual expidió Santa Cruz su decreto de disolución firmado el 20 de enero de I839.

Otra importante medida del Protector Santa Cruz fué la creación del Ministerio de Instrucción Pública, Beneficencia y Negocios Eclesiásticos por decreto protectoral, del 4 de febrero de 1837 (25). Anteriormente el ramo de educación pública estaba subordinado a otras entidades ministeriales. En I823 había dependido del Ministerio de Gobierno y a partit de 1826 había sido una dependencia de la Secretaría de Estado en el Despacho del Interior, nuevo nombre que se le dió a la anterior repartición administrativa. No se justificaba que la educación pública fuese una dependencia de un organismo político, ya que la política ha perturbado tantas veces $\mathrm{y}$ ha malogrado siempre los buenos propósitos en la marcha educacional. Si alguna explicación tienéoste erroi-ąque Santás Cruz procuró remediar acertadamente-no puede ser otra que el estado incipiente de la instrucción pública en los balbuceos desorientados de nuestro republicanismo. Santa Cruz le dió a la educación pública toda la prioridad que ella merece. Por eso organizó el Ministerio de Instrucción Pública, Beneficencia y Negocios Eclesiásticos. Por desgracia las vicisitudes políticas dejaron sin cumplimiento esta plausible disposición gubernativa que, sin embargo, quedó como un antecedente valioso en la historia de la pedagogía peruana.

(25) "El Eco del Protectorado", 8 de febrero de 1837. 
Con un claro sentido de la realidad nacional, el Protector Santa Cruz transformó el Colegio Nacional de Ciencias del Cuzco en Escuela de Artes y Oficios aprovechando y ampliando los talleres de carpintería y herreria que existían en ese plantel desde I833. En su plan de estudios, formulado con un criterio práctico, se consignaron sólo los cursos correspondientes a las artes mecánicas, reservando a 1a Universidad de San Antonio Abad la preparación de la juventud en las profesionales liberales. Nuevo y plausible esfuerzo es éste para deslindar la jurisdicción de los distintos grados de la enseñanza que tan confusos y desarticulados se presentaban entonces.

En el quinquenio I834-I839 se crearon escuelas de instrucción primaria en las provincias de Lucanas, Parinacochas y Chota. $\mathrm{Y}$ como no estaban bien atendidas las escuelas que funcionaban en los conventos, el gobierno requirió a los religiosos para que cumplieran su deber de difundir la instrucción.

No descuidó tampoco Santa Cruz la educación femenina. Consideró el Protector que la mujer "entregada hasta ahora a las mezquinas ideas que se le comunica en este departamento por un sistema aislado y restringido, no participa en los beneficios que se han procurado a los varones sin que entre tanto deje de ser de una vital importancia, poner en un mismo nivel la educación de ambos sexos en proporción a las atribuciones y deberes de cada uno". Consideró además que "el medio de conseguir tan útil objeto es establecer en esta capital un colegio de niñas educandas en el que se enseñen los rudimentos de aquellas artes y oficios que principalmente deben saber las mujeres para ser buenas madres de familia". Con tan plausible finalidad 
expidió Santa Cruz su decreto del 28 de agosto de 1838 estableciendo en Lima un colegio para niñas, bajo la protección del gobierno y la inmediata inspección del prefecto del departamento. En este plantel que funcionó en la calle del Espíritu Santo, sostenía el Gobierno treinta becas, repartidas entre las niñas de Lima y de las provincias (26).

En horas de angustiosa expectativa para la Patria, convaleciente de una guerra internacional, resquebrajadas las bases de la nacionalidad, desatado el huracán de las pasiones y de los odios, distuelto el principio de autoridad en el caos de la anarquía y cuando todo parecía confabularse contra el porvenir del Perú, dos varones insignesDomingo Elias y Nicolás Rodrigo-comprendiendo que al régimen pedagógico es la piedra angular de toda rehabilitación colectiva, fundaron el $\mathrm{I} 4$ de noviembre de $\mathrm{I} 84 \mathrm{O}$ el Colegio puesto bajo la advocación de Nuestra Señora de Guadalupe, a solicitud de Fray Juan Vargas, recordando y cumpliendo unar promesa que hizo un antecesor suyo-Fray Francisco Pérez, en la ciudad de Trujillo (México), de denominar con ese nombre de la Virgen azteca a una capilla porque, habiendo sido sentenciado a muerte a consecuencia de una calumnia, en el siglo XVIII, había sido salvado y vindicado en su honor, atribuyéndolo a una gracia del
cielo.

Gobernaba a la sazón el Perú el Mariscal Agustín Gamarra que había dado algunos decretos referentes a la instrucción pública y quien, accediendo a una solicitud de

(26) Oviedo T. 9. L. III. 
los fundadores de Guadalupe, le cedió para local del nuevo plantel un edificio de escaso valor arquitectónico, situado en la calle de Chacarilla, que había sido cuartel y había servido después para el estanco del tabaco (26 a).

El plan de estudios era sencillo. Comprendía los conocimientos de la enseñanza primaria y dictábanse además las clases de Gramática Castellana, Geografía y Matemáticas, Religión y Francés, Dibujo y Música. Colaboraba eficientemente con los directores fundadores, el vice-director Don Ramón de Azcárate, que supo imprimir acertadas orientaciones iniciales al plantel, así como una severa disciplina ( 26 b). En 1844 se amplía la enseñanza, comprendiendo entonces primeras letras y religión, Filosofía, Moral, Historia de Oriente, Matemáticas, francés, inglés, griego, latín, dibujo, música, piano, violín y clarinete. Al año siguiente se agregan los cursos de Botánica, Historia Griega, Cálculo y Mecánica.

Para darle mayor: prestigio a su Colegio Don Domingo Elías solicitó la cooperación de un eminente maestro español Dr. Sebastián Lorente quien ejercia la Cátedra de Filosofía en la Real Universidad de Madrid, quien aceptando la propuesta que se le hacía, se embarcó para el Perú. En el Colegio de Guadalupe Lorente multiplicó sus empeños y su acción constructiva; implantó un nuevo método; deste-

(26 a) "El área del terreno donde se levantaba el edificio medía 13,780 mts.2 y se emplé 7,000 pesos en su refección. En el fondo del edificio se levantaba una pileta en medio de un empedrado patio. Fronterizamente se alzaban los almacenes que sirvieron de salones a la clase elemental. El patic de estudios y recreo era enlosado. Los techos, amplios".-Revista del Centenario del Colegio de Guadalupe.

(26 b) Los profesores fundadores fueron Ignacio Agato, Mateo Ríos, Plam Batles, Miguel Coara y el célebre pintor Ignacio Merino. El primer inspector fué Apolinario Baello.-Anales del Colegio de Nuestra Señora de Guadalupe. 
rró el escolasticismo; precisó hasta donde le fué posible hacerlo los limites, entonces confusos, entre la instrucción media y el ciclo superior; le dió al plantel ese espiritu iiberal que hasta hoy mantiene como una de sus mejores tradiciones. Lo ayuda en esta empresa Pedro Gálvez quien agrega al Programa los cursos de Cálculo Infinitesimal y Astronomía.

Culmina la obra de Lorente en Guadalupe en 1847 con la organización de los estudios universitarios en el Colegio, estudiándose entonces Economía Política, Legislación, Derecho Natural, Derecho Público, Historia Natural, Gramática, Historia Moderna, Geografía, Aritmética, Geografía Física, Religión, Derecho Penal, Teneduria de Libros y Música. Al año siguiente se le agregan los cursos de Literatura, Zoologia e Historia Eclesiástica. La reforma de Lorente situó al Colegio de Guadalupe en el mismo plano pedagógico del Convictorio de San Carlos, igualdad que se consolidó en i 848 cuando 1a Universidad Mayor de San Marcos de Lima, y con ella diez uniyersidades más en América, abrieron sus puertas a los alumnos guadalupanos, declarando que los estudios realizados en ese plantel eran suficientes para discernirles el grado de Bachiller. El Gobierno del Perú, en armonía con lo informado por el Rector de la Universidad de San Marcos, otorgó valor oficial a estos estudios y ordenó que los certificados de Guadalupe surtiesen sus efectos legales. Ese mismo año, I848, la Universidad de San Marcos confirió a un alumno guadalupano el grado de bachiller.

Guadalupe y San Carlos eran ya dos planteles rivales. Estaban frente a frente y no tardaría en desatarse la lucha ideológica entre ellos. 
Los domingos de 9 a ro de la mañana se efectuaban en el Salón de Actos del Colegio disertaciones filosóficas, dirigidas por los doctores Lorente o Gálvez, quienes calificaban a los participantes. Asistían "los externos mayores y también las personas interesadas por el adelanto de la juventud" (26 c).

Un interesante documento, inédito hasta hoy, redactado por el doctor Felipe Santiago Estenós, miembro de la Junta Central de Instrucción Pública, incorporado a su Archivo Particular que obra en mi poder, arroja viva luz sobre la situación de los colegios particulares de mujeres existentes en Lima en 1850 , año que cierra, el primer período en la historia de la pedagogía republicana del Perú.

La Junta Central de Instrucción Pública, nombró una Comisión que, presidida por el doctor- Felipe Santiago Estenós (27), visitara los colegios particulares de mujeres que a la sazón funcionaban efr Lima e informara luego sobre su estado y condiciones pedagógicas.

Seis colegios de mujeres funcionaban entonces en $\mathrm{Li}^{-}$ ma: el del Espíritu Santo, el de la Encarnación, el de Concha, el Colegio Peruano, el de la Caridad y el de San An-

(27) Dr. Felipe Santiago Estenós, Secretario del Ejército Patriota expedicionario del C'entro (1822), Diputado por Arequipa en el Primer Congreso Constituyente del Perú (1822-1823), Secretario General del Libertador Bolivar (1825), Vocal fundador de la Corte Superior de Arequipa, Miembro del Consejo de Estado, Vocal de la Corte Suprema de Justicia, Miembro de la Junta Central de Instrucción Pública, Ministro del Interior durante el Gobierno de Castilla, Fiseal de la Nación, cargo en el que murió en 1864. Fué declarado Prócer de la República por ley del Congreso del Perú expedida en la Legislatura Ordinaria de 1939. 
tonio. Se dejaba sentir en todos ellos la necesidad de uniformar los textos y los métodos de enseñanza. El doctor Estenós sugeria la conveniencia de que la Junta de Instrucción examinara los textos que se estudiaban en los colegios y escogiera el mejor para adoptarlo como obligatorio para todos ellos (28).

(28) Informe presentado por el Dr. Felipe Santiago Estenós, Miembro de la Junta Central de Instrucción Pública, sobre el estado de los colegios particulares de mujeres, en Lima, en 1850.

S. S.

Lima, y Dieiembre 9 de 1850.

Al Sr. Presite. de la Junta Central de Instron. Pública.

La Comisión nombrada por la Junta pa. hacer la visita de los colegios particulares de mujeres, cumple con presentar a Ud. el resultado de la misma.

Seis son los colegios de mujeres, a saber: el del Espíritu Santo, situado en el Hospital de este nombre; el de la Enearnación, situado en la antigua Casa de Correos, próxima al Convt. de la Encarnación; el de la calle de Concha; el Colegio Peruano situado hoy en la antigua casa de Filipinas; el de la Caridad, situado en el local contiguo al que fué Hospital de este nombre, y el de la ealle de.San Antonio, cerca del Convento de la Trinidad. La Comisión extendió también su visita a-la Escuela situada dentro de la Casa de Ejercicios de Sta. Rosa.

Para facilitar la visita, eada miembro de la Comisión se eneargó de dos colegios y se exigí un estado de cada uno de ellos. El que presentó el Espíritu Santo fué el más regular y se puso de modelo a los otros pa. que se hiciesen los que habían presentado. Al fin se ha podido conseguir los que se acompaña a esta nota.

La Comisión ya reunida ha visitado esos colegios con los Estados a la vista cuidando de hacerlo a las horas de comer pa. observar el trato que se daba a las niñas y comparar también con el estado de los demás actos del Colegio. Esto ha podido hacerlo una sola vez pr. qe. Tigados sus miembros pr. las horas precisas de asistencia de los destinos que sirven, no les ha sido posible practicar la visita a diferentes horas y con la minuciosidad que *e requiere pa. poder presentar el verdadero estado de cada uno. Esta es obra de algún tiempo y era preciso presentarse en todas las clases y a diversas podis juzgar de los métodos de enseñanza, cosa que la Comisión no ha podido hacer por la razbn antedicha.

\section{Colegio del Espíritu Sto.}

El local es espacioso, los dormitorios aseados y bien ventilados, el refec. torio de bastante capacidad. La mesa se sirve por las hermanas de la Caridad, 
Caracteres de la instrucción pública durante el primer período de la ctapa republicana ( $\mathrm{I}_{2} \mathrm{I}-\mathrm{I}_{5} \mathrm{O}$ ). El confusionismo en los planes de estudio, la falta de maestros preparados, las trabas a la libertad de la enseñanza, la apatía de las autoridades, la indiferencia de los padres de familia, la escasez de las rentas escolares y la falta de organización pedagógica fueron los caracteres principales, negativos todos ellos, en este primer período en la historia de la pedagogia peruana.

ऽ también las niñas mayores hacen platos a las más chicas. Se observa orden y compostura en la hora de comer: en todo se advierte un sistema àe orden bien establecido. El alimento muy sencillo y más bien escaso que abundante. No hay más hombre en la casa qe. un sirviente para las diligencias en la calle: todo el servicio se hace pr. las hermanas de la Caridad. Todas las clases se enseñan pr. las religiosas. Los textos son los que se expresan en el Estado. El local es del Gobierno, y según noticias costea además doce becas. Hay 72 niñas internas y 16 externas. La Comisión tiene informes particulares de que la enseñanza no está bien atendida. Habría querido poder examinar esta importante circunstancia; pero no ha podido disponer de las horas necesarias.

\section{Colegio de la Encarnación.}

Situado en una casa particular de bastante extensión. Los profesores son de buena reputacion. Ni en los dormitorios, ni en las clases se nota ese sistema de orden y de aseo que resalta en el del Espiritu Santo. La comida escasa y poco atendida en su confección. Los textos sesexpresan en el Estado. Hay 57 niñas, 12 pupilas y las demás externas.

Colegio de la Calle de Concha.

Situado en una casa particular de regular extension. Los dormitorios aseados aunque no espaciosos. La comida abundante y bien condimentada. Profesores buenos. Al de caligrafía lo recomienda el Estado de este colegio pr. sus varias formas y pr. sus bellos caracteres. Es el mismo que enseña en el de la Encarnación. Los textos se expresan en el Estado. Tiene 66 niñas, 24 internas y 42 externas. El Gobierno costea tres becas.

Colegio de la Caridad.

El local es de suficiente capacidad, pertenece a la Beneficencia o al Estado: hay 4 becas gratuitas, 6 pupilas, 6 medias pupilas y 28 externas. La empresaria está recién establecida y no se notan las circunstancias que recomiendan un Colegio. Los textos pa. la enseñanza se expresan en el Estado del colegio.

\section{Colegio Peruano.}

Situado en casa particular de suficiente extension. La comida es abundante $\mathrm{y}$ bien guisada. La directora y sus hijas enseñan el mayor número de 
El confusionismo en los planes de estudio se constata tanto en la inconexión y heterogeneidad de la enseñanza en los diversos colegios como en la reglamentación distinta para cada uno de ellos. "El aprendizaje de los cursos inferiores se mezcla, sin ningún método, con el de los superiores. Enséñase, de esta suerte, simultáneamente, materias que corresponden al ciclo inferior como son aritmética, religión, teneduría de libros, geometría, historia general y eclesiástica, mecánica, latín, castellano, inglés, fran-

las clases. Una de ellas se da pr. autora del Estado que han presentado formado con buenos caracteres. Tiene 61 niñas. Los textos son los que se expresan en el Estado.

\section{Colegio de la Calle de Sn. Anto.}

En casa particular proporcionada al número de 18 niñas que son las únicas que se admiten. La Directora enseña todas las clases: está constantemente con sus discípulas y hay positivo adelanto en ellas: guardan mucha compostura en su porte, y es digna de recomendarse a la Junta la perfección conque leen y la propiedad conque pronuncian las letras. Cree la Comisión que en este pequeño Colegio las niñas aprovecharán el tiempo. Los textos de la enseñanza se expresan en el pequeũo Estado que ha presentado.

\section{Escuela de la Casa de Ejercicios.}

El local destinado a ella es un saloneito muy apropósito con bastante luz y buena ventilación. La Comisión cree que no se saca de él el provecho que era de esperarse. Faltan profesores que enseñan. La caligrafía estál atrasada y no se hace nada pr. mejorarla. A si- 10 manifiesta la letra del Estado de la escuela, que es de la niña qe. enseña a escribir. Es de necesidad me-
jorar la enseñanza.

La Comisión no puede dejar de llamar la actuación de la Junta a la diversidad de textos en estos colegios. Aunque es verdad que según el art. 40 del Rgto. de Instrucción "todo profesor puede adoptar el texto y método que mejor le parezca, previa aprobación de la Junta de Instrueción", el estado del país exige, en concepto de la Comisión, la uniformidad de los textos y de los métodos de enseñanza. El ensanche y adelanto de la conseguir también han querido consultar por el citado art. 40, se pueden Colegios, y aquel sometiendo a examen los textos que se siguen en estos todos ellos interin que resulte mejor ese debería ser el que se siguiese en

Este sistema se presentaba algún otro que fuese superior.

en el art. 20, no de los textos para la enseñanza de los ramos designados siguiente. Facilitano no perjudicaría sino que produciría el favorable efecto sabrían que sus hijos en a los padres de familia los medios de educación, de las materias que en un período de años casi fijo completarían el estudio de las materias que deben enseñarse en un colegio. Siendo los mismos en todos ellos los textos y los métodos de enseñanza, la educación de una niña no sería interrumpida como sucede al presente, cuando sus padres varían de 
cés, dibujo, música y caligrafía, confundidas todas ellas con las materias correspondientes a un ciclo superior como son Sicología, Lógica, Moral, Geometría, Trigonometría, Cálculo Infinitesimal, Astronomia, Mecánica Aplicada, Historia de la Filosofía, Estética y Derecho. No existia uniformidad en los planes de los colegios nacionales. El de Guadalupe, por ejemplo, era completamente distinto del del Cuzco. El cultivo intensivo de los idiomas implicaba, en verdad, un poliglotismo exagerado y sin control

casa a mucha distancia del colegio en que aprende pues que en eualquiera otro más inmediato a su morada, encontraria la misma enseñanza que en el que se habia visto obligado a dejar.

La Comisión considera tan necesaria una medida sobre este asunto que sin ella cree qe. progresará el mal que hace en el día la variación en textos y métodos no solo de colegio a colegio, sino dentro de ellos. Se varían los textos según se varían los profesores, lo que trae consigo la neesidad de volver a empezar los cursos. Esto hace perder el tiempo y ocasiona a las familias gastos considerables; y como uno de los modos de proteger la enseñanza es facilitar los medios de alcanzarla, lo cual está en la esfera de las atribueiones de la Junta Central. a tenor de la 9a. de las facultades que le dá el art. 31 del Reglamento, la C'omisión somete este punto a su ilustrada consideración.

Coadyuvando a este sistema de uniformidad la Comisión juzga conveniente reducir los colegios de niñas a la enseñanza de las materias que prescribe el art. 20 del Rgto., suprimiendo las clases que se enseñan demás y obligando a establecer las que falten en la actualidad. No hay cosa que pueda oponerse a esto y pr. otra parte es un deber que a la Junta Central le impone el Rgto. Según el art. 20 debe enseñarse en los colegios de niñas "dibujo, música, toda especie de costura llana, deshilado, bordados, tejidos y demás obras manuales propias de su sexo, reglas de urbanidad, moral y cconomía doméstica, gramática castellana, aritmética, francés, inglés, geografía descriptiva, breves nociones de historia general, reglas de higiene privada y religión".

La Comisión considera de necesidad que la Junta ordene: que el primer dibujo que se enseña a las niñas sea el de ramificación, sin cuyo conocimiento no deben pasar al bordado pa. que ellas mismas dibujen lo que han de bordar, como parece natural y como lo exige la urgente necesidad de cortar el abuso de cobrar a las niñas por separado el dibujo del bordado. Ninguna niña debe pasar a la clase de bordado mientras que sus adelantamientos en la de dibujo de ramificación no faciliten los trabajos preparatorios de aquella. Después đel dibujo de ramificación pueden pasar al natural. Este y otros abusos semejantes se remediarán uniformando los colegios de niñas según el trascrito art. $20 \mathrm{y}$ obligando a todos a la publicación del programa de enseñanza que previene el art. 39 como regla invariable del contrato que liga a las directoras con los padres que les confían a sus hijas. 
$y$, por lo mismo, perjudicial, ya que los altumnos en vez de aprender bien un solo idioma, aprendían muy mal varios de ellos y, por ende, no aprovechaban de ninguno. Los cursos de latín, inglés y francés figuran siempre en los planes de los colegios. Algunos de ellos se incrementan, además, con el italiano y con el griego. El latín, sobre todo, campeaba en todos los ciclos de la enseñanza, desde la primaria hasta la superior, a tal punto que en este idioma debían ser pronunciados, en la colación de grados académicos de la Universidad de San Marcos, las tesis de los graduados.

El aseo de las niñas en los colegios se debe considerar como una parte importante de su educación, lo mismo que el cuidado que cada una de ellas debe tener de conservar en buen orden todas sus cosas de colegio. De este ramo de edueación tan esencial a las que han de ser madres de familia, no se tiene el esmerado cuidado que requiere. Influye en el atraso de la enseñanza pr. el desaliento qu inspira al repetido gasto que demanda a los padres la continua reposición de las cosas que se pierden o destruyen. Como los colegios de niñas son de empresa particular pr. cuanto aunque algunos son auxiliados por la Nación con el local y con algunas becas, ninguno es costeado por ella, la Comisión cree que la Junta no puede ejereitar su acción benéfica más allá de lo relativo a la enseñanza y educación, pues qe. en lo demás los padres deben euidar de las mejoros condiciones eonque sus hijas deben ser tratados. Si los padres celan esta parte de los colegios, como está en sus intereses hacerlo, y retiran a las niñas del en que las traten mal, este será un estímulo poderoso pa que mejoren su condición. Para esto es de absoluta necesidad que los padres tengan la entrada franca a fin de poder examinar los tratos que reciban sus hijas en las distribuciones del colegio y ver si los directores cumplen con las obligaciones contraidas pa. con cada una de ellas. En el Colegio del Espíritu Sto. es vedado esto y los padres son obligados a pasar por lo que se les dice que se hace o se harí. En este colegio no salen las niñas a sus casas sino al primer domingo del mes o una vez al mes. Si esto es bueno en cuanto contribuye a arraigar los principios y los hábitos que practican en el Colegio no lo es desde que los directores no tienen con las niñas el cuidado de su madres pa. asearles el cuerpo como debieran hacerlo. No lo hacen, según está instruída la Comisión y las niñas son víctimas del desaseo. Es pues de necesidad qe. salgan los domingos. Con esta medida se evitará también la mortificación que sufren las madres de poeas proporciones, en los dias festivos qe. les es per: fomenta la competencis en el colegio, no pudiendo presentarse con el lujo que cia.

La Comisión debe llamar la atención de la Junta sobre un hecho que ha verificado con las mismas actores, $y$ es que las niñas se han de confesar precisamente con el C'apellán del Colegio, que es un sacerdote francés, sin que a los padres les sea permitido elegir el confesor pa. sus hijas. 
La diversidad de los planes producía, como es natural, la multiplicidad de reglamentos escolares. Cada colegio. tenía su reglamento propio (29).

La falta de maestros idóneos era otro de los males de que adolecía la enseñanza. No se preocupaban los maestros de entonces en conocer la sicología del niño. Quienes aspiraban a ejercer un cargo magisterial, más por necesidad aue por vocación, estaban obligados a rendir un examen de meros conncimientos sobie la materia de su enseñanza ante un Turado Oficial aue los declaraba antos $O$ inentos según los casos. Un decreto expedido en la época de Gamarra nrescribía el concurso nara los asnirantes al título. mediante un examen que debía durar dos horas ante 11n Turado que emitía su fallo en votación secreta $(30)$. El Perí. en materia de maestros, salvn rarísimas excenciones. vivía con un siglo de atraso en relación a Eurona. Nuestro panorama era idéntico a aquel otro aue en el Viejo mundo; a principios de la pasada centuria impugnó con energía Pestalozzi, el gran reformador de la enseñanza en la magnífica experiencia de Ivefdón, a orillas cel Lago Nenemburg. Eran generalmente esos maestros gentes sin voca-

Si la Junta acoge favorablemente las indicaciones de la Comisión y resuelve el arreglo de las cosas según propone; la visita se acordará de modo permanente para celar cada uno de los actos del Colegio en sus clases $y$ fuera de ellas con la atención y sagacidad que tienen derecho a esperar unas señoras que en la ocupación honrosa qe. han abrazado hacen un servicio importante al país. Los colegios se aumentarán a proporción de los provechos que reporten las emvresarias $y$ pa. que sean activos $y$ mayores pueile la Junta propender a ahorrar los gastos fuertes que hacen pa. presentar los exámenes públicos a qe. están obligados. Estos gastos se evitarán destinándose y habilitándose por el Gobierno un local apropósito donde todos los colegios presentan sus exámenes.

Dios gue. a V. S.

(fdo.) Felipe Santiago Estenós.

(29) “El Mensajero de Tacna”.-Semanario.-N.e 14, del sábado 18 de abril de 1840 .

(30) "El Correo Peruano".-Primer semestre.-17 de febrero de 1840. 
ción, que percibian sueldos misérrimos, dedicados al trabajo memorista y a una labor puramente mecánica e imitativa, sin preocuparse por el desarrollo mental y físico de los niños, sin interés en estimular la natural espontaneidad y viveza en los alumnos y su entusiasmo por el aprendizaje y sin comprender toda la trascendencia social de la función educadora.

Las trabas a la libertad de enseñanza restringieron también considerablemente sus perspectivas. El más acérrimo impugnador de la libertad de enseñanza, en esta época, fué el Ministro José Gregorio Paz Soldán. En su circular del 18 de diciembre de 1845 previno a los Rectores de las Universidades, por intermedio de los Prefectos, para inpedir que en los grados académicos se sostengan tesis contrarias a las regalías del Patronato Nacional, so pena de declararse nulas. Especificaba, además, que las actuaciones debían estar "arregladas en el Derecho Natural a las doctrinas de Ahrens, Felipe Burlamaqui y Heinecio; etl el de Gentes, a las de José María Pando, Bello, Wattel, Kluber, Martens, Piñeyro y Reinabal; en lo civil, a las leyes patrias; y en el canónico a lo que enseñan Pereyra, Van Espén, Berardi, Cavalario, Cañada y Cobarrubias. Dos años después, en I847, persistiendo en sus mismos conceptos, el Ministro Paz Soldán dirige una nueva circular a los Prefectos de los Departamentos, ordenándoles que supervigilaran la enseñanza en los colegios de su jurisdicción, cuiclando de que se estudiaran los textos recomendados; que se haga de memoria el estudio sobre todos los cur- 
sos; que en los exámenes públicos se pongan sobre las mesas, además de las tablas, los textos en los que se han estudiado las materias del examen; y que "en la asignatura del Derecho Canónico se sostengan de todos modos las regalías del Patronato Nacional y las libertades de la Iglesia peruana, sin permitirse la enseñanza de doctrinas que directa o indirectamente puedan dañar lọ derechos de la Nación, su independencia y soberanía".

La apatía de las autoridades encargadas de la instrucción contribuía a agravar el mal. No existían, en realidad, autoridades especializadas en el ramo. Eran los funcionarios políticos a quienes subsidiariamente se encargaba de las cuestiones educativas para las que no estaban preparados y por las que, en consecuencia, no sentian ningún celo. Algunos Ministros, más por plataforma política que por interés pedagógico, intentaban innovaciones muchas veces inoficiosas y casi siempre prematuras, sin esperar los resultados de los anteriores ensayos, tratando de deshacer en no pocos casos lo que se había hecho durante el gobierno precedente. Continuas ordenes ${ }^{\prime} y^{\prime \prime}$ contra-órdenes entrababan aún más, por eso, la acción de las autoridades locales.

La indiforencia $d c$ los propios padres de familia no era menos punible que la apatía de las autoridades escolares. No se preocupaban los padres, por lo general; de la educación de sus hijos. La realidad, en este sentido, no había variado desde la época bolivariana en que la enjuició el prócer Sánchez Carrión. Al promediar el siglo pasado todavía tenía toda su dolorosa actualidad la frase del $\mathrm{Mi}^{-}$ nistro de Bolívar expresada en el Congreso Nacional. Todavía, como él lo afirmara en 1825 , los padres se negaban a 
mandar a sus hijos a las escuelas y las autoridades se desentendían de estos y de otros cuidados semejantes.

Entonces, ahora y siempre, el problema económico ha sido una de las bases fundamentales para el progreso educacional. Sin las rentas adecuadas han de fracasar todos los buenos propósitos de la reforma pedagógica. No podía ser más deplorable la escasez de las rentas escolares en este primer período de la historia de la pedagogía republicana. La pobreza del erario público invalidaba todo intento de superación educativa. Se cometió entonces el error de creer que las escuelas podían sostenerse con fondos locales que generalmente estuvieron muy mal administrados, siendo objeto alguna que otra vez de punibles malversaciones. En la Circular que Don Manuel Carpio dirige a los Prefectos en 1845 les manifiesta que "es deplorable el estado de penuria a que se hallan reducidos los preceptores de las aulas de instrucción que paga el gobierno y esto procede del escandaloso atraso que hay en las cobranzas de las rentas de estélraino"e Un periódiéo limeño criticaba en I848, "el estado de deçadenicialỳ de desgreño en que se hallan los fondos dedicados a la instrucción pública en todos los pueblos del Perú, porque se malversan impunemente las entradas dedicadas para este objeto, no existiendo en los pueblos las escuelas necesarias".(3I).

La falta de criterio pedagógico campeaba también entonces en las actividades de la educación nacional y era otra de las causas de su decadencia. No se tenía en cuenta-y así lo comprueban los horarios, ciclos de estudio, etc.la necesidad de los descansos reglamentarios. No se organizaba un rol apropiado de exámenes promocionales, en-

(31) "El Zurringo", N.9 1, del 11 de marzo de 1848. 
tregándolos por completo a la iniciativa de cada uno de los colegios. De ahí que unos planteles verificaban sus pruebas en diciembre, otros en enero, algunos en marzo. Casi todos ellos las desarrollaban íntegramente en un plazo máximo de cinco días lo que era realmente agobiador para el alumnado por el esfuerzo intelectual que este sobretrabajo les demandaba. Tampoco se veían libres las Universidades de anomalías semejantes, sobre todo en la colación de grados, confiriéndolos, en no pocas ocasiones, previa dispensa de las pruebas de suficiencia, sólo con exámenes de Derecho Natural y de Gentes, lo que motivó la intervención del Ministro de Instrucción Don José Gregorio $\mathrm{Paz}$ Soldán quien, por circular del 5 de julio de 1845 , previno a los Rectores de las Universidades y Presidentes de Cortes para que reprimiesen estas corruptelas.

El periodismo de la época refleja la decadencia de la instrucción pública y sus anhelos de subsanarla. Quejándose de este abandono, aboga "El Zurriago", en uno de sus editoriales, porque "la instrucción se propague en todas las masas, desde la "Tldea mảs pobre hasta tos espléndidos palacios de la capital porque sólo así es posible tener ciudadanos morales, instruídos y capaces de conocer sus derechos" (32).

El periódico arequipeño "Pabellón Nacional" pide al Congreso que "se digne considerar que siendo la instrucción pública una de las condiciones más indispensables $\mathrm{pa}^{-}$ ra el progreso de nuestra sociedad, debe procurar no sólo

(32) "El Zurriago", N.॰ 7 del 19 de abril de 1848. 
dar leyes que jamás se cumplen para favorecer su desarrollo, sino también adoptar medidas seguras para que el Ejecutivo no se contente con la charla estéril que a este respecto suele estampar en sus documentos, sino para que real y verdaderamente proteja la ilustración primaria $y$ perfeccione la educación científica de la juventud". Y agrega: "Ruboriza decir en presencia de los extranjeros que habitan esta ciudad que en Arequipa no hay una escuela regular y medianamente decente de primeras letras". Refiriéndose a la Universidad de Arequipa, reclama: "Algo más que pedimos es que cualquiera no pueda ser maestro de una facultad sin que se le cén las cátedras por concursos a aquellos que poseen vastos conocimientos y no a uno de los mismos alumnos que no tienen el tiempo preciso para contraerse al ramo que estudia como sucede frecuentemente entre nosotros en ciertas facultades" (33).

$\mathrm{El}$ advenimiento al poder del Mariscal Castilla en 1845 , después de uno de los ciclos más agitados y turbulentos de nuestra vida independiènte abre una nueva era, caracterizada por los felices intentosedecsistematización, en la historia de la pedagogía peruana.

\section{Roberto Mac-Lean y Estenós.}

(33) "Pabellón Nacional", N. ${ }^{\circ} 37$, editado en Arequipa, año 1848. 